EXTENDED REPORT

\title{
Anterior scleral canal geometry in pressurised (IOP 10) and non-pressurised (IOP O) normal monkey eyes
}

\author{
A J Bellezza, C J Rintalan, H W Thompson, J C Downs, R T Hart, C F Burgoyne
}

Br J Ophthalmol 2003;87:1284-1290

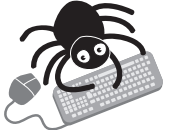

Additional tables can be found on the BJO website www.bjophthalmol.com/ supplemental

See end of article for authors' affiliations

.....................

Correspondence to:

C F Burgoyne, MD, LSU

Eye Center, 2020 Gravier

Street, Suite B, New

Orleans, LA 70112, USA;

cburgo@|suhsc.edu

Accepted for publication 15 January 2003

\begin{abstract}
Aims: To characterise lamina cribrosa and anterior scleral canal wall architecture in pressurised (IOP $10 \mathrm{~mm} \mathrm{Hg}$ ) and non-pressurised (IOP $0 \mathrm{~mm} \mathrm{Hg}$ ) normal monkey eyes.

Methods: Eight normal eyes from eight monkeys were enucleated before sacrifice and the optic nerve heads (ONH) trephined and immersion fixed in glutaraldehyde (IOP 0). Nine normal eyes from nine monkeys were perfusion fixed in situ with paraformaldehyde at IOP $10 \mathrm{~mm} \mathrm{Hg}$ (IOP 10), and the ONHs trephined and stored in glutaraldehyde. Each $\mathrm{ONH}$ specimen was embedded in glycol methacrylate and cut into vertical or horizontal, $4 \mu \mathrm{m}$ thick, serial sections. Within digitised images of every sixth section, anterior laminar position and laminar thickness were measured at nine evenly spaced locations across the scleral canal opening. Additionally, scleral canal diameters at Bruch's membrane (SCD-B) and at the anterior laminar insertion (SCD-ALI) were measured within the 15 middle section images of each vertically sectioned $\mathrm{ONH}$.

Results: Anterior laminar position was significantly more anterior (nearer Bruch's membrane) in the IOP 10 eyes, compared with the IOP 0 eyes $(116( \pm 95 \% \mathrm{Cl} ; 2) \mu \mathrm{m} v 184(2) \mu \mathrm{m}$, respectively). Also in the IOP 10 eyes, the lamina cribrosa was thinner (195 (2) $\mu \mathrm{m} v 264(2) \mu \mathrm{m})$ and the scleral canal diameter was larger (SCD-B: 1751 (23) $\mu \mathrm{m} v 1591$ (19) $\mu \mathrm{m}$; SCD-ALl: 1961 (21) $\mu \mathrm{m} v 1717$ (17) $\mu \mathrm{m})$, compared with the IOP 0 eyes.

Conclusion: The anterior scleral canal wall is expanded and the lamina cribrosa is thinned and more tautly stretched within pressurised (perfusion fixed at IOP 10) young monkey eyes, compared with nonpressurised (immersion fixed at IOP 0) young monkey eyes. The constricted scleral canal and the relaxed and thickened lamina in the non-pressurised eyes may represent phenomena that contribute to optic disc swelling in hypotonous eyes.
\end{abstract}

$\mathrm{O}$ ptic nerve head $(\mathrm{ONH})$ surface compliance testing, which has been performed in several laboratories, ${ }^{1-3}$ is based on the detection of posterior deformation of the $\mathrm{ONH}$ surface following acute elevations of intraocular pressure (IOP). Such IOP induced deformations of the ONH surface are presumed to follow deformations of the underlying connective tissues of the lamina cribrosa, scleral canal wall, and peripapillary sclera. ${ }^{4-6}$ However, until now, the ability to characterise the position and thickness of the lamina cribrosa within the scleral canal has been limited, and a rigorous study of the three dimensional architecture of the lamina at varying levels of IOP has not been performed.

As part of our larger efforts to characterise the $\mathrm{ONH}$ as a biomechanical structure, we are currently studying the mechanical behaviour of the load bearing connective tissues of the peripapillary sclera, scleral canal wall, and lamina cribrosa in normal and early glaucomatous monkey eyes. To demonstrate the responses of these tissues to the mechanical stresses generated by varying levels of IOP, we have immersion fixed and perfusion fixed a large group of normal and early glaucomatous monkey eyes at IOPs of $0,10,30$, or $45 \mathrm{~mm} \mathrm{Hg}$ and serially sectioned each eye at $4 \mu \mathrm{m}$ intervals in either a vertical or horizontal sagittal direction.

In this report, we use the techniques described above to study anterior scleral canal architecture in two groups of normal, young adult monkey eyes: nine eyes of nine monkeys perfusion fixed at IOP $10 \mathrm{~mm} \mathrm{Hg}$ and eight eyes of eight monkeys immersion fixed at IOP $0 \mathrm{~mm} \mathrm{Hg}$. Parameters measured include lamina cribrosa position relative to Bruch's membrane, laminar thickness, and scleral canal diameter at Bruch's membrane opening and at the anterior laminar insertion of each eye. The results suggest that for young adult monkeys, there are marked differences in anterior scleral canal architecture in pressurised (IOP $10 \mathrm{~mm} \mathrm{Hg}$ ) and non-pressurised (IOP $0 \mathrm{~mm} \mathrm{Hg}$ ) eyes.

\section{METHODS}

\section{Animals and study eyes}

All animals were treated in accordance with the ARVO resolution on the use of animals in ophthalmic and vision research. Anterior scleral canal architecture was characterised within digital images from serial sagittal sections of the $\mathrm{ONH}$ from one eye each of a total of 17 male rhesus and cynomolgus monkeys with estimated ages ranging from 4.3 to 11.1 years (Table 1 ). Nine normal eyes from nine monkeys were perfusion fixed with glutaraldehyde at a normal IOP of $10 \mathrm{~mm} \mathrm{Hg}$ (IOP 10). Eight normal eyes from eight monkeys were enucleated before sacrifice, and their $\mathrm{ONH}$ tissues were immersion fixed in glutaraldehyde at an IOP of $0 \mathrm{~mm} \mathrm{Hg}$ (IOP 0).

\section{Perfusion fixed IOP 10 eyes}

Our method for perfusion fixation has been previously described. ${ }^{7}$ Under deep pentobarbital anaesthesia, both eyes of nine monkeys were cannulated with a 27 gauge needle, and IOP was set to $10 \mathrm{~mm} \mathrm{Hg}$ in each eye using an adjustable saline reservoir. As part of a separate experiment, 15 minutes before perfusion fixation, IOP was elevated to either 30 or $45 \mathrm{~mm} \mathrm{Hg}$ in one eye, with the other (study) eye maintained at IOP $10 \mathrm{~mm} \mathrm{Hg}$. Each monkey was then perfusion fixed via 


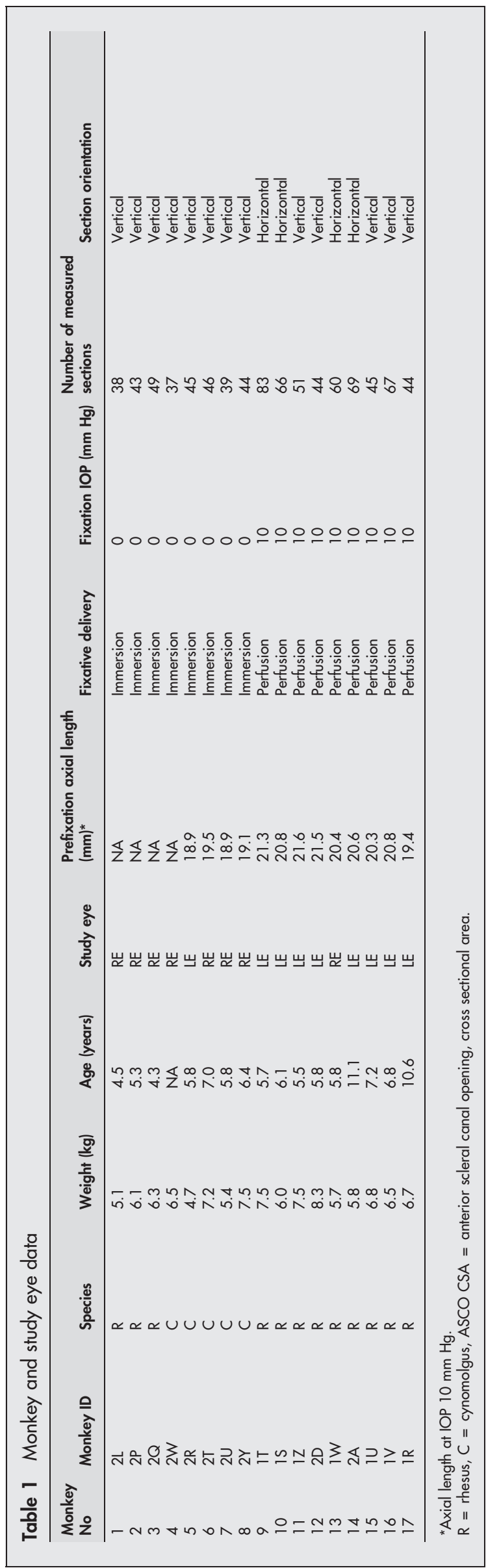

the descending aorta with 1 litre of $4 \%$ buffered hypertonic paraformaldehyde solution followed by 6 litres of 5\% buffered hypertonic glutaraldehyde solution. Following perfusion, IOPs were maintained for 1 hour, after which the eyes were enucleated, all extraorbital tissues were dissected away, and the anterior chambers were removed $2-3 \mathrm{~mm}$ posterior to the limbus. By gross inspection, perfusion was excellent in all of the IOP 10 eyes. The posterior scleral shells, with choroid and retina intact, were stored in $4 \%$ glutaraldehyde solution. The ONH and peripapillary sclera of the normal IOP $10 \mathrm{~mm} \mathrm{Hg}$ eye of each monkey was later isolated from the posterior scleral shell by passing a $6 \mathrm{~mm}$ trephine from the retina through the sclera.

\section{Immersion fixed IOP 0 eyes}

Our method for immersion fixation has been previously described. ${ }^{7}$ Briefly, under deep pentobarbital anaesthesia, both eyes of eight monkeys were enucleated, opened posterior to the limbus, and placed into chilled normal saline. Each monkey was then sacrificed with an intracardiac dose of pentobarbital. For each eye, a $6 \mathrm{~mm}$ diameter trephine was used to separate the $\mathrm{ONH}$ and peripapillary sclera from the posterior scleral shell by cutting from the outside of the sclera towards an underlying polypropylene ball. The trephined $\mathrm{ONH}$ tissue was placed into buffered, hypertonic 5\% glutaraldehyde solution, and the remainder of the unfixed posterior scleral shell was processed for other studies.

\section{ONH specimen preparation and sectioning}

At the time of processing, one edge of each specimen was trimmed for either horizontal (four of the perfusion fixed IOP 10 eyes) or vertical (five of the perfusion fixed IOP 10 eyes and all eight of the immersion fixed IOP 0 eyes) sectioning. The ONH surface of each specimen was photographed with the scleral canal edge of the retinal pigment epithelium in focus, along with a companion image of a slide mounted micrometer scale, to generate an estimate of the post-fixation cross sectional area of the anterior scleral canal opening at Bruch's membrane. The specimens were then dehydrated, infiltrated, embedded in historesin (Technovit 7100; Kulzer, Wehrheim, Germany), and sagittally sectioned at $4 \mu \mathrm{m}$ intervals (model RM2165 microtome, Leica, Bensheim, Germany). Histological sections were mounted on glass slides, stained with Van Gieson's stain, and cover slipped.

\section{Section image acquisition and measurement parameter generation}

Our method for image acquisition and measurement has also been previously described. ${ }^{7}$ For each $\mathrm{ONH}$, the anterior scleral canal opening was defined to coincide with the opening in Bruch's membrane. This definition was chosen because Bruch's membrane is easily identified within histological sections and clinically visible (with some degree of variability) within clinical fundus images. Using this definition, the first and last histological section in which Bruch's membrane was intact established the boundaries of the anterior scleral canal opening. The number of sections measured for each $\mathrm{ONH}$ varied depending on the size of the opening and whether the sections were cut vertically or horizontally (Table 1).

A composite image of every sixth sagittal section was generated as described previously (Fig 1)..$^{7}$ Each image was processed using a custom image analysis program as follows: (1) Bruch's membrane opening, the anterior laminar insertion, and the anterior and posterior lamina cribrosa position were identified by an operator masked to the treatment 


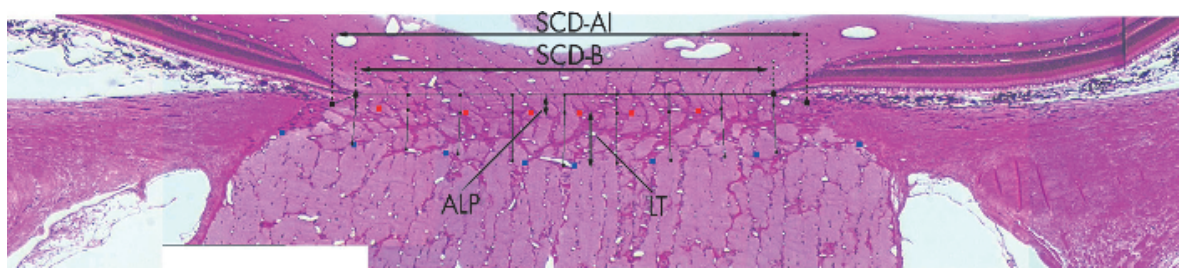

Figure 1 Generation and measurement of composite section images. Each composite section image consists of four to six individual overlapping images taken at a resolution of approximately $2 \mu \mathrm{m} /$ pixel. On each section image, an operator placed marks denoting the termination of Bruch's membrane, the anterior insertion of the lamina into the sclera, and the anterior and posterior border of the lamina cribrosa. A custom image analysis software then connected the two Bruch's membrane termination points, divided the distance into nine measurement points, and dropped perpendicular lines to the anterior and posterior lamina cribrosa as best determined by the image analysis software based on the operator's landmarks. Next, nine automatic measures of anterior laminar position (ALP) and laminar thickness (LT) across the scleral canal opening were made, as well as one measurement of the diameter of the scleral canal opening at Bruch's membrane (SCD-B) and at the anterior laminar insertion (SCD-ALI). (Adapted from Bellezza et alp.)

condition of the image being marked; (2) anterior lamina cribrosa position and lamina cribrosa thickness were automatically measured at nine evenly spaced locations across the anterior scleral canal opening; (3) anterior scleral canal diameter was automatically measured at both Bruch's membrane and the anterior laminar insertion; and (4) data for each measurement parameter were saved for each image to a spreadsheet file (Excel; Microsoft, Redmond, WA, USA) for each $\mathrm{ONH}$.
For each ONH, all images were marked by one of two operators (CJR and AJB) who were masked to the treatment group of that $\mathrm{ONH}$. All images for an $\mathrm{ONH}$ were available to the operator, and frequent study of sections "before" and "after" the section being marked was often necessary to best place the marks. After all images for an ONH were marked, a single operator (AJB) reviewed each image to ensure overall consistency of the marks. The reproducibility of our method has previously been reported. ${ }^{7}$

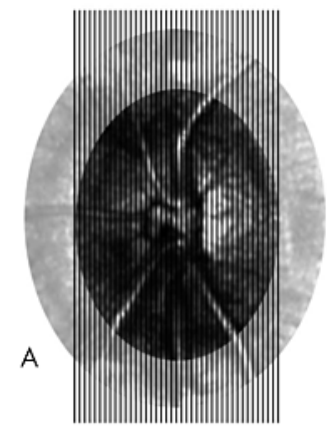

Monkey 12 , LE

44 vertical section images

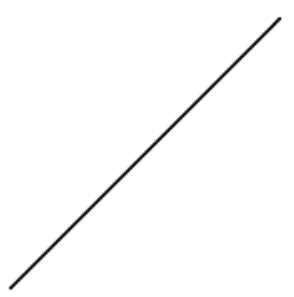

Laminar position and thickness

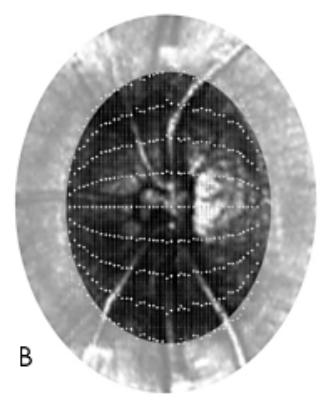

Overall

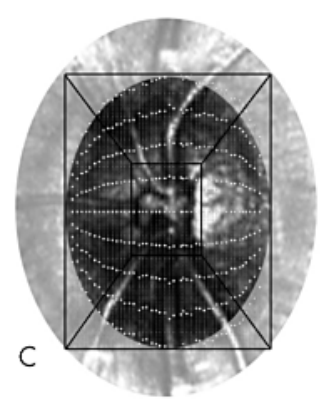

Regional

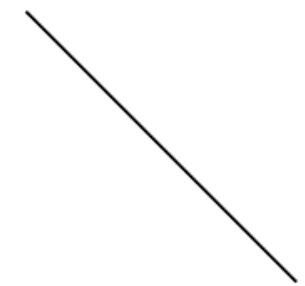

Scleral canal diameter

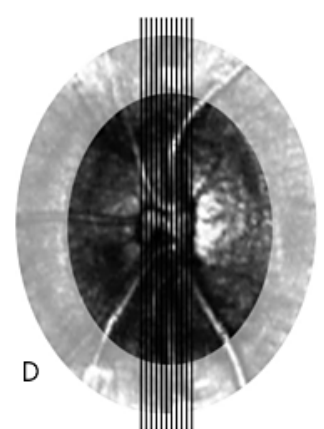

Middle 15 sections
Figure 2 Example of pooling the data for an individual optic nerve head. (A) Measurements were made on the set of images taken of the sections cut from between the termination points of Bruch's membrane. (B) The nine measurement points for anterior laminar position and thickness (see Fig 1) from each section image were projected onto a confocal scanning laser tomographic image of the measured optic nerve head. (C) The measurement points were subdivided into central, superior, inferior, nasal, and temporal regions (see methods for details). (D) Vertical scleral canal diameter and anterior scleral canal wall geometry measurements were made only within the middle 15 section images. (Adapted from Bellezza et alp.) 

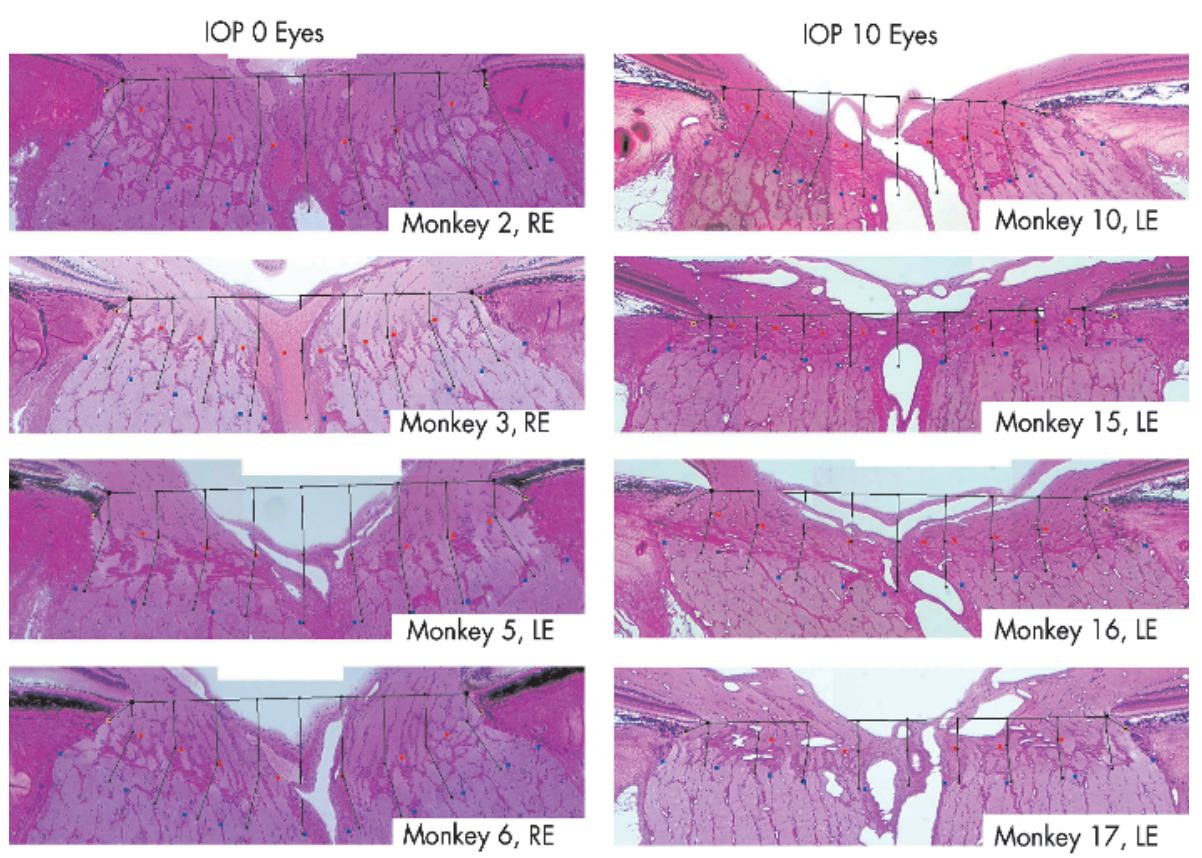

Figure 3 Representative middle sagittal section images from four representative IOP 0 and four representative IOP $10 \mathrm{ONH}$ s. Note that within the IOP 0 eyes (left), the lamina cribrosa appears "sagged" posteriorly relative to the "taut," more anteriorly positioned lamina in the IOP 10 eyes

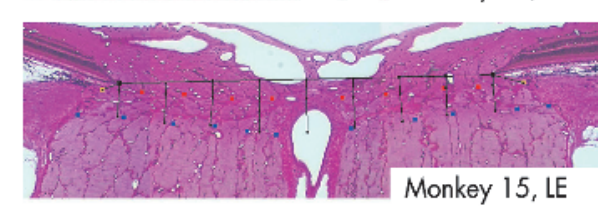
(right).

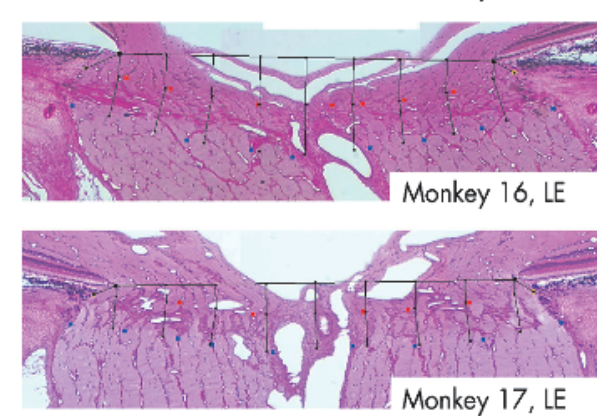

\section{Overall and regional anterior laminar position and laminar thickness}

Within each image, anterior laminar position and laminar thickness were measured at nine points which, taken across all measured images, created a grid of measurement points that covered the entire scleral canal opening. For each of the $17 \mathrm{ONH}$ specimens, the grid of measurement points was superimposed onto a confocal scanning laser tomographic image of the ONH surface that was obtained before sacrifice (Fig 2A B). Each point was then assigned to one of five regions: central (centred on the central vascular tree), superior, inferior, nasal, or temporal (Fig 2C). Regions were determined by surrounding the scleral canal opening with a rectangle, then creating a central region boundary one third the height and width of the outer box. The central region was positioned to best encompass the central vasculature, and varied from one $\mathrm{ONH}$ to another. By connecting the corners of the inner and outer boxes, the four peripheral regions were constructed. Mean values for anterior laminar position and laminar thickness were calculated for each specimen overall (all points combined) and for each of the five regions separately.

\section{Anterior scleral canal diameter}

For determination of the scleral canal diameter, only measurements from the middle 15 section images for each vertically sectioned eye were used (Fig 2D). Mean anterior scleral canal diameter was calculated at Bruch's membrane (Fig l, SCD-B) and at the anterior laminar insertion (Fig l, SCD-ALI).

\section{Statistical analysis for differences between treatment groups \\ Lamina cribrosa position and thickness}

A nested ANOVA was used to assess the effects of region (central, superior, inferior, nasal, and temporal) and treatment group (IOP 0 versus IOP 10) on the parameters anterior lamina cribrosa position and laminar thickness.

Anterior scleral canal diameter

A separate ANOVA was used to assess the effects of treatment group on anterior scleral canal diameter. In this analysis, two treatment groups were assessed: vertically sectioned IOP 10 ONHs ( $\mathrm{n}=5$ eyes), and vertically sectioned IOP 0 ONHs $(\mathrm{n}=8$ eyes).

Comparisons between treatments, regions, and treatment by region combinations were made using protected $t$ tests between least squares means in which the $\mathrm{p}$ values were adjusted for the number of multiple comparisons made. ${ }^{8}$ All $p$ values reported are derived from these tests. In addition to the hypothesis tests performed with the ANOVAs, magnitudes of all measured parameters were assessed by computation of $95 \%$ confidence intervals using least squares means. ${ }^{9}$ All results are given as a mean plus or minus its $95 \%$ confidence interval.

\section{RESULTS}

Individual monkey and study eye data are shown in Table 1 . Representative vertical sagittal sections for four of the IOP 0 and four of the IOP 10 eyes can be seen in Figure 3.

\section{Comparison of lamina cribrosa position and thickness} Overall and regional lamina cribrosa position and thickness are reported in Table 2. Overall, the lamina cribrosa was both more anterior and thinner in the IOP 10 eyes, compared with the IOP 0 eyes $(\mathrm{p}<0.0001)$ (anterior laminar position, 116 $( \pm 95 \%$ CI 2) $\mu \mathrm{m} v 184$ (2) $\mu \mathrm{m}$; laminar thickness, 195 (2) $\mu \mathrm{m} v 264$ (2) $\mu \mathrm{m}$, IOP 10 eyes $v$ IOP 0 eyes, respectively).

Table 2 Lamina cribrosa position and thickness by IOP group*

\begin{tabular}{llllll}
\hline & \multicolumn{2}{l}{ Anterior laminar position } & & \multicolumn{2}{l}{ Laminar thickness } \\
\cline { 2 - 3 } \cline { 5 - 6 } Region & IOP 10 & IOP 0 & & IOP 10 & IOP 0 \\
\hline Overall & $116(2)$ & $184(2)$ & & $195(2)$ & $264(2)$ \\
Central & $161(4)$ & $267(6)$ & & $223(4)$ & $268(4)$ \\
Superior & $102(2)$ & $151(4)$ & & $179(2)$ & $273(4)$ \\
Inferior & $106(4)$ & $158(4)$ & & $186(2)$ & $270(2)$ \\
Nasal & $109(4)$ & $181(4)$ & & $189(2)$ & $258(4)$ \\
Temporal & $102(2)$ & $162(4)$ & & $199(2)$ & $252(2)$ \\
\hline
\end{tabular}

*Values are means plus or minus $95 \%$ confidence intervals in $\mu \mathrm{m}$ 
Table 3 Vertical scleral canal diameter by IOP group*

\begin{tabular}{lll}
\hline & $\begin{array}{l}\text { IOP 10 (mm Hg) } \\
(\mathbf{n}=5)\end{array}$ & $\begin{array}{l}\text { IOP O (mm Hg) } \\
(\mathbf{n}=8)\end{array}$ \\
\hline $\begin{array}{l}\text { Bruch's membrane } \\
\text { Anterior laminar } \\
\text { insertion }\end{array}$ & $1751(23)$ & $1591(19)$ \\
$1961(21)$ & $1717(17)$
\end{tabular}

*Values are means plus or minus $95 \%$ confidence intervals in $\mu \mathrm{m}$.

Similar differences were also present within each of the five scleral canal regions $(p<0.0001)$.

By region, the relative posterior displacement (sagging) of the lamina in the IOP 0 eyes was greatest centrally (anterior laminar position, 267 (6) $\mu \mathrm{m} v 161$ (4) $\mu \mathrm{m}$, IOP 0 eyes $v$ IOP 10 eyes, respectively). However, differences in laminar thickness between the IOP 10 and IOP 0 eyes were least in the central region and greatest in the periphery (superior, inferior, nasal and temporal regions) (Table 2). Laminar position and thickness values for each of the IOP 0 eyes are provided in Table 1 on the BJO website, and for each of the IOP 10 eyes in Table 2 on the BJO website.

\section{Anterior scleral canal diameter}

Anterior scleral canal diameters at Bruch's membrane and at the anterior laminar insertion are reported in Table 3. Vertical scleral canal diameter at both Bruch's membrane and the anterior laminar insertion was significantly larger in the IOP 10 eyes, compared with the IOP 0 eyes $(\mathrm{p}<0.0001)$ (Bruch's membrane, 1751 (23) $\mu \mathrm{m} v 1591$ (19) $\mu \mathrm{m}$; anterior laminar insertion, 1961 (21) $\mu \mathrm{m} v 1717$ (17) $\mu \mathrm{m}$, respectively).

\section{DISCUSSION}

The principal findings of this report are as follows. Firstly, the vertical anterior scleral canal opening at both Bruch's membrane and the anterior laminar insertion in normal young adult monkey eyes is substantially larger in pressurised (IOP 10) eyes than in non-pressurised (IOP 0) eyes. Secondly, the lamina cribrosa is thinner and is pulled anteriorly in the pressurised eyes.

To explain these findings, we propose the notion of an expandable trampoline (Fig 4) and suggest that in the "relaxed" IOP 0 eyes, the canal itself is unstretched, and the lamina cribrosa sags backward within the canal. Once pressurised, however, even at a pressure of $10 \mathrm{~mm} \mathrm{Hg}$, the scleral canal wall is pulled outward by the tensile forces within the peripapillary sclera and the laminar beams are pulled taut within the canal, demonstrating a net anterior movement of the lamina towards the scleral canal opening at Bruch's membrane.

These findings support the notion that the $\mathrm{ONH}$ is a dynamic structure in which the load bearing connective tissues of the peripapillary sclera, scleral canal wall, and lamina cribrosa dynamically respond to the mechanical stresses generated by varying levels of IOP. Our data additionally suggest that the magnitude of the response at low levels of IOP is substantial. Two important clinical implications follow from these observations.

Firstly, the compliance of the lamina cribrosa and anterior scleral canal wall at low levels of IOP should vary with and be a manifestation of the relative rigidity of the tissues. There is evidence to suggest that the compliance of the lamina decreases with age. Albon et $a l^{6}$ demonstrated that in older eyes, there was not only a decrease in posterior deformation of the lamina following an acute increase in IOP relative to that seen in younger eyes, but there was also a lessened ability to recover to the original state when IOP was decreased. In a study of 22 children who underwent IOP lowering surgery, Quigley ${ }^{10}$ observed that $40 \%$ of those whose surgery was successful had an improvement in the appearance of their optic disc cup, suggesting a resilience in younger patients' $\mathrm{ONH}$ connective tissues. An age related change in compliance is most likely the result of changes in the extracellular matrix components of the lamina cribrosa and scleral canal wall. ${ }^{11}{ }^{12}$ These changes may include an increase in the amount and density of collagen types I, III, and IV, ${ }^{11}$ as well as an increase in the crosslinking of elastin with other proteins, which has been implicated in the age related stiffening of elastic tissues. ${ }^{12}{ }^{13}$

Zeimer $^{14}{ }^{15}$ hypothesised that a lamina cribrosa that is stiffer than its accompanying scleral canal is more susceptible to damage than a lamina with viscoelastic material properties that better match its surrounding canal wall. In this theory, the less stiff scleral canal wall would want to expand further than the stiffer lamina cribrosa in response to an IOP increase. Because of this, the lamina is forced to stretch beyond its elastic limits to match the expansion of the more pliable scleral canal wall, potentially damaging the laminar beams at their point of insertion.

Secondly, the "sagged" state of the lamina in the unpressurised eyes may explain the clinical phenomenon of optic disc swelling at low IOP. In the pressurised state (even at an IOP of $10 \mathrm{~mm} \mathrm{Hg}$ ), the lamina is pulled taut within the scleral canal, and one would expect each laminar pore to be slightly expanded as a result. Conversely, in the unpressurised state, laminar pore distension may diminish, causing a secondary compression of laminar capillaries and peripheral axon bundles. Minckler $e t a l^{16}$ reported some degree of axonal transport blockage at the lamina in normal eyes and an increase in axonal congestion in low IOP eyes. Clinically, hypotony is commonly associated with swelling of the $\mathrm{ONH}$ and is thought to be an important risk factor in seriously damaged eyes that lose vision following glaucoma filtration surgery.

We propose the following scenario for the overall mechanical behaviour of these tissues. Firstly, as IOP increases from $0 \mathrm{~mm} \mathrm{Hg}$, the sclera and, within it, the scleral canal opening, expand. Under the predominant influence of the tensile stresses produced by the scleral canal expansion, the laminar
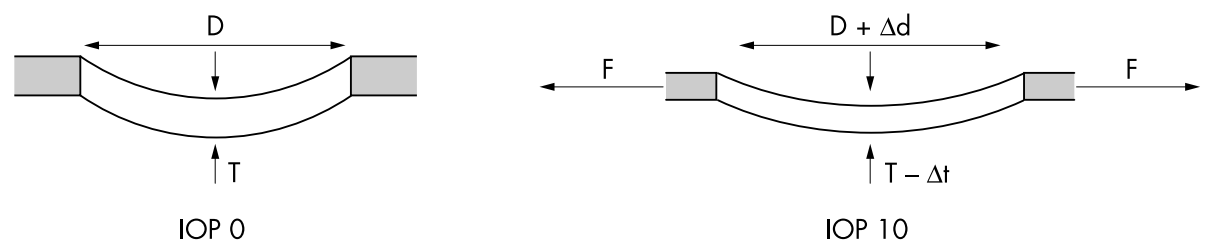

Figure 4 Schematic representation of the lamina cribrosa and scleral canal in a non-pressurised (IOP 0) and pressurised (IOP 10) eye. Left: Thickness (T) of the lamina cribrosa and diameter (D) of the scleral canal opening in an unpressurised (IOP O) eye. Right: Pressure within the globe generates an expansion of the scleral shell which, in turn, generates (and is resisted by) tensile forces within the sclera. These forces (F) act on the scleral canal wall, causing the scleral canal opening to expand $(\Delta d)$, which in turn stretches the lamina within the canal. Thus, the lamina is taut (more anteriorly positioned) and thinned ( $\Delta t$ ) in the IOP 10 eye, compared with the IOP 0 eye. 
cribrosa is pulled taut within the canal and moves forward to a maximally anterior position relative to the scleral canal opening at Bruch's membrane. This initial pressurisation serves to align the unstretched collagen and elastin fibres within the lamina and scleral canal wall. In this low level pressurised state, the size of the intralaminar fenestrations would be increased and axonal transport least affected by physical obstruction due to external compression by the laminar trabeculae.

At some level of IOP, the amount of scleral canal expansion resulting from a given amount of IOP elevation begins to lessen. This phenomenon is the likely result of a transition from straightening kinked collagen fibres to stretching fibres which are now fully distended. From this point, further IOP elevation begins to deform the already taut lamina posteriorly from its most anterior position as the effects of laminar tensile stress (pulling the lamina tight within the canal) are exceeded by the posterior bending forces generated by the direct effect of intraocular pressure pushing the prelaminar ONH surface back within the scleral canal.

Zeimer and Edward, ${ }^{17}$ using postmortem human eyes subjected to an acute increase in IOP, reported a decrease in $\mathrm{ONH}$ displacement associated with an increase in posterior scleral canal diameter. Additionally, their study showed a correlation between lamina cribrosa thinning and scleral canal diameter enlargement, supporting our scenario that the lamina cribrosa is pulled taut and thinned in concert with scleral canal expansion.

Our data suggest that the thickness of the lamina cribrosa in the normal monkey eye (overall, $195 \mu \mathrm{m}$ for the IOP 10 eyes and $264 \mu \mathrm{m}$ for the IOP 0 eyes, Table 2 ) is comparable to that of its human counterpart. Quigley and associates ${ }^{18}$ reported an average laminar thickness of $237 \mu \mathrm{m}$ in 12 normal human donor cadaver eyes. Yan and co-workers ${ }^{5}$ measured laminar thickness in a single central section of 10 normal human cadaver eyes fixed at a pressure of $5 \mathrm{~mm} \mathrm{Hg}$ and found the average thickness to be $121 \mu \mathrm{m}$. Interestingly, in their study, significant thinning of the lamina was not detected in the individual central sections from the 10 contralateral eyes fixed at $50 \mathrm{~mm} \mathrm{Hg}$.

Three aspects of this study need to be considered. Firstly, because the conditions under which the eyes used in these studies were obtained were dictated in part by the requirements of other studies, the results are based on differences in scleral canal diameter between monkey eyes immersion fixed at IOP 0 and monkey eyes perfusion fixed at IOP $10 \mathrm{~mm} \mathrm{Hg}$. The drawback here is that the position of the tissues at no pressure may depend on many unknown factors, including fixation, enucleation, sectioning, and so forth. It might have been preferable to compare changes between a positive pressure and a more physiological pressure such as $15 \mathrm{~mm} \mathrm{Hg}$, but other requirements of our work did not allow this.

Secondly, because the sclera of the IOP 0 eyes was used for a separate study which required unfixed tissues, the ONHs of the IOP 0 eyes were trephined before fixation in a direction (from the outside of the eye through to the retina) opposite that of the IOP 10 eyes, and then immersion fixed rather than perfusion fixed. It is possible that the difference in trephine direction, or the fact that the ONH tissues were trephined before fixation in the IOP 0 eyes, may account for our findings. A study in which both eyes of an individual monkey are perfusion fixed-one at IOP 0 and the other at IOP 10 -is necessary to confirm these differences between pressurised and non-pressurised eyes and is currently under way in our laboratory.

Thirdly, both the paraformaldehyde and glutaraldehyde fixation solutions used in this study were hypertonic. We are unsure of the effect a hypertonic fixative may have on the
ONH tissues. However, fixation appeared to be excellent in both groups of eyes and the effect is likely to be consistent across the specimens. We thus believe that the relative differences between groups are unlikely to be related to fixation hypertonicity.

In summary, our results suggest that the connective tissues of the anterior scleral canal in normal young adult monkeys are surprisingly compliant under the range of IOP related stress generated between IOP 0 and $10 \mathrm{~mm} \mathrm{Hg}$. The results further suggest that contraction of the scleral canal and relaxation of the lamina cribrosa are present at low levels of IOP and may contribute to the pathophysiology of hypotonous optic disc swelling.

\section{ACKNOWLEDGEMENTS}

This work was supported in part by USPHS grants R01EY11610 (CFB) and P30EY02377 (departmental core grant, LSU Eye Center) from the National Eye Institute, National Institutes of Health, Bethesda, Maryland; a grant from the American Health Assistance Foundation, Rockville, Maryland (CFB); a grant from The Whitaker Foundation, Rosslyn, Virginia (CFB); a Career Development Award (CFB) and an unrestricted departmental grant (LSU Eye Center) from Research to Prevent Blindness, Inc, New York, New York; and a graduate student stipend from the Board of Regents, State of Louisiana (AJB). The authors wish to thank Stephanie Hager, Budd Hirons, Juan Reynaud, and Lindell Skinner for their invaluable technical assistance.

\section{Authors' affiliations}

A J Bellezza, H W Thompson, J C Downs, C F Burgoyne, LSU Eye Center, Lovisiana State University Health Sciences Center, New Orleans, LA, USA

A J Bellezza, C J Rintalan, J C Downs, R T Hart, C F Burgoyne, Department of Biomedical Engineering, Tulane University, New Orleans, LA, USA

This work was presented in part at the Association for Research in Vision and Ophthalmology Annual Meeting. Fort Louderdale, Florida, May 2001.

\section{REFERENCES}

1 Zeimer RC, Ogura Y. The relation between glaucomatous damage and optic nerve head mechanical compliance. Arch Ophthalmol 1989;107:1232-4.

2 Coleman AL, Quigley HA, Vitale S, et al. Displacement of the optic nerve head by acute changes in intraocular pressure in monkey eyes. Ophthalmology 1991:98:35-40.

3 Quigley HA, Pease ME. Change in the optic disc and nerve fiber layer estimated with the Glaucoma-scope in monkey eyes. J Glaucoma 1996;5:106-16.

4 Levy NS, Crapps EE, Bonney RC. Displacement of the optic nerve head. Response to intraocular pressure elevation in primate eyes. Arch Ophthalmol 1981;99:2166-74.

5 Yan DB, Coloma FM, Metheetrairut A, et al. Deformation of the lamina cribrosa by elevated intraocular pressure. Br J Ophthalmol 1994;78:643-8.

6 Albon J, Purslow PP, Karwatowski WS, et al. Age related compliance of the lamina cribrosa in human eyes. Br J Ophthalmol 2000;84:318-23.

7 Bellezza AJ, Rintalan CJ, Thompson HW, et al. Deformation of the lamina cribrosa and anterior scleral canal wall in early experimental glaucoma. Invest Ophthalmol Vis Sci 2003;44:623-37.

8 Edward D, Berry JJ. The efficiency of simulation-based multiple comparisons. Biometrics 1987;43:913-28.

9 Milliken GA, Johnson DE. Analysis of messy data. Volume I: Designed experiments. New York: Van Nostrand Reinhold, 1984:29.

10 Quigley HA. Childhood glaucoma: results with trabeculotomy and study of reversible cupping. Ophthalmology 1982;89:219-26.

11 Hernandez MR, Luo XX, Andrzejewska W, et al. Age-related changes in the extracellular matrix of the human optic nerve head. Am J Ophthalmol 1989; 107:476-84

12 Albon J, Karwatowski WSS, Easty DL, et al. Age related changes in the noncollagenous components of the extracellular matrix of the human lamina cribrosa. Br J Ophthalmol 2000;84:311-17.

13 Schofield JD, Weightman B. New knowledge of connective tissue ageing. J Clin Pathol (Suppl) 1978;12:174-90.

14 Zeimer R. Biomechanical properties of the optic nerve head. In: Drance SM, Anderson DR, eds. Optic nerve in glaucoma. Amsterdam: Kugler Publications, 1995: 107-21.

15 Zeimer R. Could glaucoma damage be due to a viscoelastic mismatch between the sclera and the lamina cribrosa? J Jap Glaucoma Soc 1992;2:17-20. 
16 Minckler DS, Tso MO, Zimmerman LE. A light microscopic, autoradiographic study of axoplasmic transport in the optic nerve head during ocular hypotony, increased intraocular pressure, and papilledema. Am J Ophthalmol

1976;82:741-57.
17 Zeimer $\mathbf{R}$ Edward DP. Structural changes in the lamina cribrosa associated with glaucoma. Glaucoma 1992;14:182-6.

18 Quigley HA, Hohman RM, Addicks EM, et al. Morphologic changes in the lamina cribrosa correlated with neural loss in open-angle glaucoma. Am J Ophthalmol 1983;95:673-91.

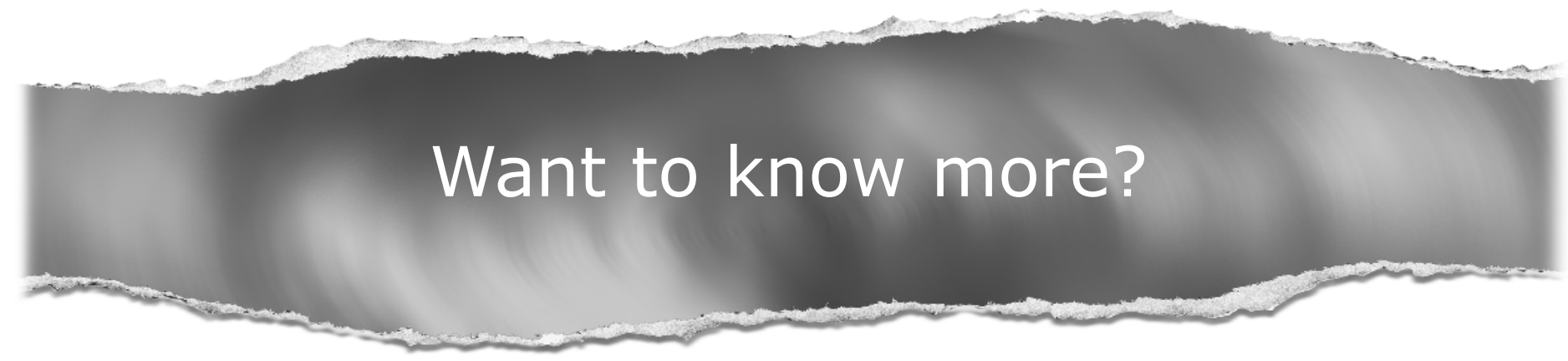

Data supplements

Limited space in printed journals means that interesting data and other material are often edited out of articles; however, limitless cyberspace means that we can include this information online. Look out for additional tables, references, illustrations, and (soon to appear) multimedia clips online!

\section{www.bjophthalmol.com}

